\title{
RESEARCH ARTICLE \\ RESEARCH ON THE DANCE ENTERTAINMENT SYSTEM BASED ON USER EXPERIENCE
}

\author{
Tang Qilin \\ Hunan University of Technology, China
}

This is an open access article distributed under the Creative Commons Attribution License, which permits unrestricted use, distribution, and reproduction in any medium, provided the original work is properly cited

\section{ARTICLE DETAILS}

\section{Article History:}

Received 1 February 2019 Accepted 18 March 2019 Available online 22 March 2019

\section{ABSTRACT}

Dance, a culture form of entertainment, has the value of art and appreciation, at the same time it plays the role of physical fitness, thus it becomes a kind of cultural behavior that is highly welcomed by people now. However, there are various types of dance with different forms, and the same kind of dance may bring different feelings to the audience. On the basis of experience of users, empirical analysis method was used in this paper, so the current situation of the present dance entertainment system in our country was analyzed and the existing problems were found out, and the reasons were put forward. Corresponding strategies to improve the design were proposed too. Experimental results show that which has theoretical and practical significance.

\section{KEYWORDS}

User experience, dance entertainment system, strategy improvement

\section{INTRODUCTION}

Experience of users refers to the subjective formation of the feelings and evaluation of a product or a service when the customer uses such products or services. Although it is a subjective feeling, now the user experience has been quantified by technology [1]. A large number of user experience measurements are from the beginning of the experience, which means that using the relevant models to divide the user experience factors in order to facilitate the analysis [2]. The American economist Joseph uses two coordinates to divide the user experience, which is shown in Figure 1, and the two coordinates happen to divide the user experience into four modules and the modules contact with each other, constituting the dimensions of the user experience [3].

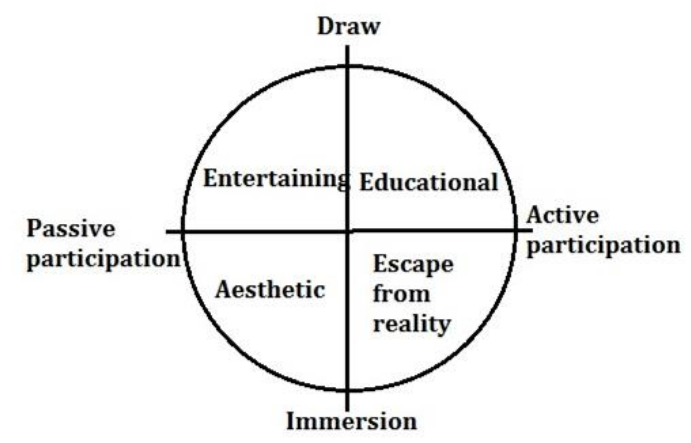

Figure 1: Pine Experience Classification

Dance entertainment system refers to the digital integrated system which is composed of various media forms which are related to the dance and can react with the dance program [4]. And it is the application of a new technology in the dance. About dance entertainment system, domestic and foreign scholars have carried out a lot of researches [5]. Although there is not many researches about dance entertainment system based on user experience and now the domestic research results have not formed a complete system, many theories associated with user experience can be able to communicate with the user experience of dance entertainment. Now, some of the analysis for user experience of many experts in our country is carried out in a wide variety of applications, such as evaluations of user experience in a web site, user experience evaluation of mobile phone software and user experience evaluations of children software [6]. Although in the field of dance entertainment system research results of user experience are still lack [7]. However, thought and logic of user experience researches are intrinsic interlinked. Therefore, theoretical arguments proposed in this paper are not directed against the dance entertainment system, but they are equally applicable to the research ideas of user experience [8].

In this paper, the research methods used are literature analysis method and empirical analysis method, and the relevant researches of domestic dance entertainment system are investigated on-the-spot and data is collected, and on the basis of this, the models are established for mathematical analysis. On the research ideas. Firstly, this paper summarizes and present status of the dance entertainment system researches and finds out the existing problems, then it analyses the causes of the problems, finally according to the reasons, the corresponding solving measures are put forward in the view of user experience.

\section{STATE OF THE ART}

At present, the dance entertainment products of traditional dance entertainment system generally have three forms: somatosensory entertainment dance, dance blanket dance entertainment and mobile tablet dance entertainment [9]. These three kinds of forms have a mutual relationship. 


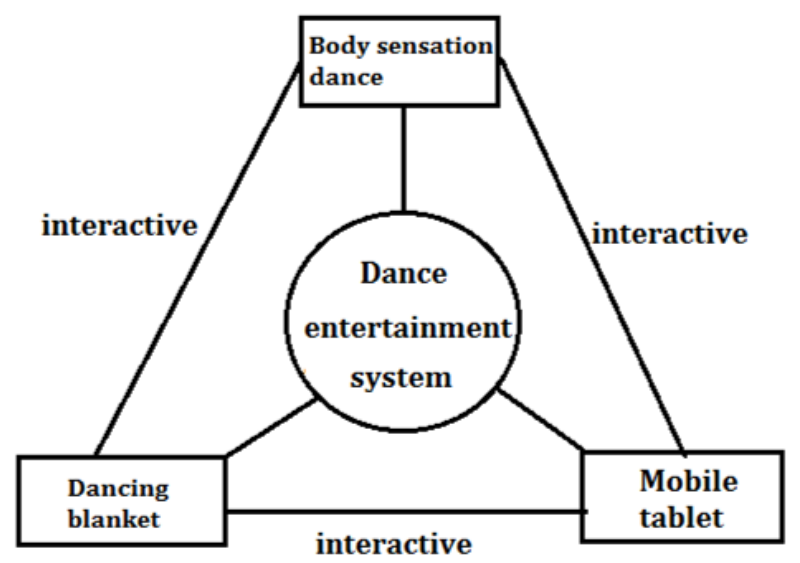

Figure 2: Dance entertainment system content structure

Dancing blanket is the simplest form of dance entertainment. After the starting of accompaniment, users only need to correspondingly step to the arrow icons on the feet in accordance with the continuous scrolls of the screen [10]. If the signs that the user steppes on are different from the arrows, the screen will show the user is wrong; if the user marks the arrows correctly, you can get through the checkpoints. The benefits of this form of dance entertainment are that it is not subject to site constraints and it is simple and convenient, but the disadvantage is that its organs confined to the legs and feet and there are no activities for the user's body, so the user experience is just general [11]

Mobile tablet dance entertainment system is the operation and experience of entertainment software downloaded by the players, and users need to hand held electronic devices, for example, Pad. The gravity sensing device of mobile phones and tables can judge the direction of movement of the limb of the user and it also can analyze whether the users make a behavior of squat [12]. This dance entertainment system is not so convenient compared with dancing blanket, and the sensitivity of the response actions is also very poor and the accuracy of the actions of the judge is relatively rough, so it is difficult to achieve a truly excellent user experience.

Somatosensory dance is substantial physical dance, this entertainment does not require participants to wear any equipment, and it only requires the participants to launch the body joints following one's inclinations and specific actions of dancing can be different according to the different situations of different design [13]. Somatosensory dance entertainment system is a kind of forms of entertainment that are close to the nature and it is a kind of real dance, so the participants can feel simple, natural and smooth and in the whole process they have pleasure and immersed sense, but this form is limited by the professionalism of participants, so the implementation of breadth is not high.

It is not difficult to see that the current problem of the dance entertainment system is that many entertainment forms of dancing cannot achieve the user experience perfectly. Due to the limitation of professional quality, not everyone can have dance training in the field [14]. Therefore, the traditional dance entertainment system must fully consider the importance of user experience based on the original basis and design a suitable dance entertainment system for the current users, and the specific design process is discussed below.

\section{METHODOLOGY}

\subsection{Design method and procedures of dance entertainment system}

Design of dance entertainment system uses the approach of "goal orient", which refers to the "dual target" of users and designers. Namely, the designer in the design of a dance entertainment product must consider the audience's psychology and behavior patterns, and when they are thinking into their own thoughts, they also take the ideas if customers into account, so as to design the products with the dual goals.

Designing the oriented goal of the dance system has a total of six steps: first of all, make the market research. The designer studies the products, markets and users; secondly, build the product model, which means modeling; thirdly, define requirements; then make the definition and construction of the whole framework; and then refine the overall framework that has been established; finally, support the establishment of the work. The six steps are closely linked, and any problems of one part will affect the final results and the whole process is accompanied by a feedback and checking, as shown in Figure 3

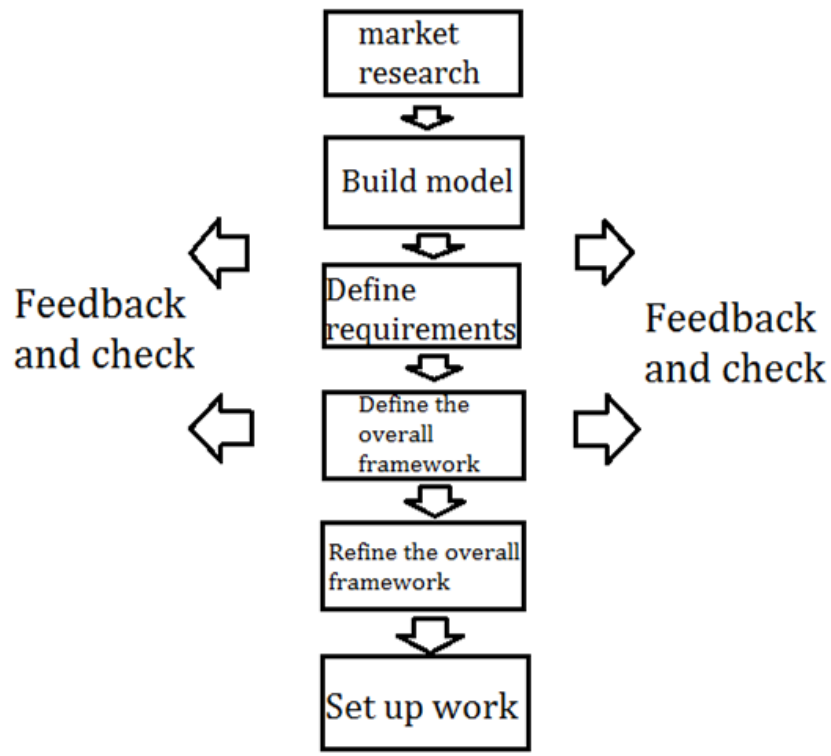

Figure 3: Steps to create a dance entertainment system

\subsection{Demand analysis of dance entertainment system}

Demand analysis is the key step in the design of dance entertainment system, this step is a preparation in front of all the work, so the designer must consider the following issues: Who is the audiences of dance entertainment system, what do the users of dance entertainment system want to get, how the system works, and what forms can achieve the effects in the operation. These questions are very basic but very important, and it is must be grasped by the designer. Most users or participants have their own preference on dance. However, more and more people do not make direct dance entertainment activities because of the limitation of time. In addition, some moves of dance is difficult to achieve for the participants who do not have the professional training. Therefore, the dance entertainment system based on the user experience must include instructions and learning contents of dance and action tests, and the focus is to let users and dance entertainment system have natural interactions, reducing the cognitive load of the participants in the interaction process.

\subsection{Interactive design of dance entertainment system based on user experience}

Interactive design, which is alternate design, and its main task is to conduct the behaviors of the design of artificial system. And the interactive design of dance entertainment system based on user experience gives consideration to the user experience in the development of dance entertainment products as far as possible, and if the interactive process is done well, the improvement of user experience can be ensured. This design process mainly includes three parts, they are the interaction design of main interface, Exit menu interaction design and teaching dance interaction design.

First, making the user design of main interface. The dance entertainment system is usually composed of a free mode and a teaching mode, so the main interface must include the user's choice between the two modes. For these two models, you can take the right hand to choose. The gestures of right and left hand can mostly be seen in the unlock of entertainment system and the open behaviors in films and televisions, so most users will have a psychological inertia, namely, they put their hand in fingerprint location that will open a certain behavior. Therefore, using the double metaphor of the door shape and fingerprints can help users choose the mode and enhance the user experience.

Second, making user experience design of Exit menu. The dance entertainment system must set up the function that users can exit the 
current section and return to the previous interface in each of its subordinate modules. This feature requires the users to complete by drawing a cross on the chest with the arm, the reason is that the cross means stop in the life. In this way, a connection is established between the dance entertainment system and real life, which fully considers the user experience.

Finally, designing the teaching dance. Since the user must always learn to move, so the error operation is very easy to happen. This time we need to carry out two stages of design: First of all, make decomposition on the dance actions, which will become a consecutive series of tiny movements, so the participants only need to learn the small action based on each basic dance. Finally, connect all the actions. After the end of the action of the participants, the system will make identification and scoring. If the scores are less than 60 points, the next action will not be carried out, if the scores are more than 60 points, the users can carry out the next action. This kind of dance with the nature of the assessment of learning can fully mobilize the enthusiasm of the participants with incentives, so it is a good basis for the user experience.

\subsection{Visual design of dance entertainment system}

This article takes the peacock dance as an example to discuss the entertainment system design. Peacock dance is an action that is an extremely graceful dance art, and it is the most representative, identified and extensive dance in Dai ethnic dance, at the same time, it is a national dance with the highest degree of recognition and visibility. Visual design of dance is a very important part, including the contents of the scene, visual design, visual interface design, visual design and animation visual design.

In the visual scene design, based on the user experience, it should be able to reflect the Dai ethnic customs. And because the Dai people are long-term living in the mountains, so the tones of the scene design need to contain natural scenery of Yunnan Province. At the same time, the building with characteristics of Dai can be put in the scene. In interface design, color is very important, in this case, the color of the interface uses the blue green, showing the nature, blue sky and trees, and the color chosen echoes the color of the peacock. In personage's visual design, we should pay more attention to the character of folk costumes through reduction design, and characters of dance entertainment system use yellow and blue as the main color, so under the background of green, figures can be extruded and the screen can also be lively. In animation design, we should pay attention to the accurate performance of information, at the same time, the aesthetic and emotional performance is also very important, so in the dance teaching animation, first of all the dance should be accurately set, and then adjusting the lens push-pull and shot cut, at last, the special efficiency is produced. In summary, the entire dance entertainment system will maximize the user experience to take the mobilization into account.

\section{RESULT ANALYSIS AND DISCUSSION}

User experience is critical to the product, which determines the quantity and quality of the product. Based on the user experience, this chapter analyzes the measurement methods, the process and the results of the user experience of the dance entertainment system, and it analyzes the prospects of the application of the entertainment system.

\subsection{Usability evaluation of dance entertainment system}

The usability evaluation of dance entertainment system mainly consists of two aspects: performance evaluation and evaluation of degree of satisfaction. Degree of satisfaction of users reflects the degree of satisfaction of the user's psychological and emotional needs of the entertainment system. The performance evaluation reflects the influence of the system design on the user's use efficiency. This paper takes 10 students from Nanjing Industrial University as an example, and in separate rooms the users completely and independently study the first teaching dance and the second learning action of peacock dance. We shoot the whole process and post processing data. When the users complete the experience of peacock learning dance and free dance, they will complete a satisfaction questionnaire.

First of all, make performance evaluation. In this study, the time of completing the peacock learning dance with dance entertainment system and the total number of operations and errors in the operation are made into statistics, the results are shown in table 1 :

Table 1: User dance learning results statistics

\begin{tabular}{|c|c|c|c|c|}
\hline Participant & Time taken for tests (second) & Action learning time (second) & Error operation times & Total operation times \\
\hline 1 & 126 & 59 & 1 & 4 \\
\hline 2 & 110 & 62 & 0 & 3 \\
\hline 3 & 130 & 48 & 1 & 4 \\
\hline 4 & 152 & 76 & 2 & 5 \\
\hline 5 & 109 & 52 & 0 & 3 \\
\hline 6 & 113 & 47 & 0 & 3 \\
\hline 7 & 146 & 89 & 3 & 5 \\
\hline 8 & 131 & 78 & 2 & 5 \\
\hline 9 & 128 & 68 & 1 & 4 \\
\hline 10 & 118 & 56 & 0 & 3 \\
\hline Average & 126.3 & 63.5 & 1 & 3.9 \\
\hline
\end{tabular}

The rate of operation errors of the system, time of unit action learning and completion rate of effective tasks are calculated. As shown in table 2: 
Table 2: System error operation rate, unit action learning time and effective task completion rate

\begin{tabular}{|c|c|c|c|}
\hline Title & System error operating rate & Unit action learning time & Effective task completion rate \\
\hline Average & $25.60 \%$ & 31.8 & $100 \%$ \\
\hline
\end{tabular}

From the results in Table 2, it is not difficult to find that the operating rate of system errors is relatively high, and in the post analysis, the sources of error has two, on the one hand, participants unconsciously clasp their hands while using the system, so the system automatically returns to a last level. On the other hand, in the course of operation, the position of the system has been moved, which affects the subsequent identification of the system to the users. In addition, some of the experimental participants driven the other operations in the curiosity. Time-consuming of unit action learning and completion rate of efficient tasks are entirely consistent with expectations, and in dividing the unit dance moves, time of duration of each action is roughly in seven to ten seconds, but it also ensures the continuity of motion. Learning time of unit action of users of performance test is about 31.8 seconds. This result is a level with high learning conversion rate. Therefore, it can be said the system in the detection of availability performance achieves good results.

Secondly, make evaluation of user satisfaction. We conduct a questionnaire survey whose full mark is 5 on the existing problems and conduct a questionnaire survey of users who use the dance entertainment system, and the results were shown in the following table:

Table 3: The peacock dance entertainment system user satisfaction scale

\begin{tabular}{|c|c|c|}
\hline Subject & Questions & Average \\
\hline \multirow{4}{*}{ Aesthetic experience } & 1 propaganda animation to bring the feeling of beauty & 4.8 \\
\hline & 2 teaching animation reflects the elegance of the dance. & 4.5 \\
\hline & 3 free dance module brings me the feeling of Dai dance & 4.6 \\
\hline & Average value of aesthetic experience & 4.63 \\
\hline \multirow{4}{*}{ Congnitive load } & 1 system operation is easy to understand & 4.8 \\
\hline & 2 animation in the dance way is easy to learn & 4.6 \\
\hline & 3 use the system to feel relaxed & 4.7 \\
\hline & Average score of cognitive load & 4.7 \\
\hline \multirow{4}{*}{ Interactive experience } & 1 system information feedback in a timely manner & 4.5 \\
\hline & 2 system information feedback accurately & 4.9 \\
\hline & 3 system interaction design makes me feel happy & 4.5 \\
\hline & Average score of interactive experience & 4.3 \\
\hline
\end{tabular}

It is not difficult to find that the average value of the degree of satisfaction of users is 4.54 , and the users agree that the identity system design has a good interactive experience, aesthetic experience and a lower cognitive load, and in some details, there are still surviving spaces.

In summary, from the results of the comprehensive performance test and user satisfaction test, the usability of the design of the dance entertainment system is still relatively high.

\section{CONCLUSIONS}

User experience is very important for any kinds of products, and it affects the quality of products and customer evaluation. Dance entertainment system is an extremely important part of entertainment system of today's society, if we want to let the traditional dance entertainment system make innovation and constantly adapt to the society, we must carry on analysis and improvement based on user experience. The content of this article is relatively simple, and the theory is not accurate enough, but in the followup study of the scholars, we believe that the theory of this field will be more and more perfect.

\section{REFERENCES}

[1] Tong, W. 2015. Design and user experience of dance entertainment system based on natural interaction. Journal of Harbin Institute of Technology, 2015

[2] Li, Z.Y., Yi, M.L. 2013. A summary of the research on website optimization based on user experience. information science, 11(5), 11-14.

[3] Deng, S.L., Hu, C.P. 2006. Based on the user experience of the website information construction elements and model analysis. information science, 3 .

[4] Liu, B. 2012. Reflection and interpretation of information quality based on the perspective of user experience, library and information work, 6.

[5] Liu, B., Lu, S. 2011. Study on information quality comprehensive evaluation system based on user experience. library and information work, 22.

[6] Wang, S. 2015. The Chinese dance in the consumption era. comments, 2015.06

[7] Tong, Y. 2005. China on value orientation of contemporary dance. Journal of Beijing Dance Academy, 3 
[8] Cui, S.Y. 2014. Modern society and high level entertainment dance. Shanghai artist, 1994.05

[9] Dong, Y. 2012. China's fitness function of dance sports culture guide system of, 2.

[10] Sun, J. 2012. The function and characteristics of social dance. group of literary world, 11(4), 13-44.

[11] Liu, C. 1999. New media of dance. Journal of Beijing Dance Academy,
4.

[12] Lin, C., Hu, J. 2012. Hole quality (QoE) model and evaluation method of user experience quality, Journal of computers, 1(3): 13(2), 22-52.

[13] Li, X.Q. 2010. User experience design based on user psychology research. Information science, 5 .

[14] Sun, X.H., Liu, J. 2011. What determines the full experience of the user of the product. psychological science progress, 1.

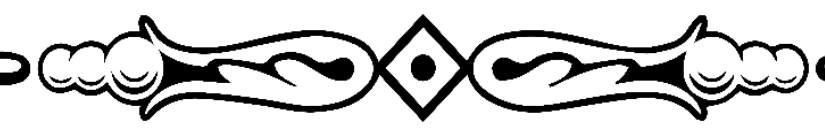

